\title{
PROPOSED METHOD FOR HANDOVER IN MOBILE NETWORK USING FUZZY LOGIC
}

\author{
Sudha P.N. ${ }^{1}$, Chanda.V. Reddy ${ }^{2}$ \\ 'Department of Telecommunication Engineering, K.S.Institute of Technology, Bangalore, Karnataka, India \\ ${ }^{2}$ Department of Electronics \& Communication Engineering, \\ K.S.Institute of Technology, Bangalore, Karnataka, India \\ E-Mail :'pnsudha@gmail.com
}

\begin{abstract}
Processing handoffs is an important task in any cellular radio system. Handoffs must be performed successfully and as infrequently as possible, and be imperceptible to the users. In order to meet these requirements, system designers must specify an optimum signal level at which initiate a handoff. Once a particular signal level is specified as the minimum usable signal for acceptable voice quality at the base station receiver, a slightly stronger signal level is used as a threshold at which a handoff is made. This margin, given by $\Delta=\mathrm{Pr}_{\text {handoff }}-\mathrm{Pr}_{\text {minimum usable, }}$ cannot be too large or too small [1]. Therefore, $\Delta$ is chosen carefully to meet these conflicting requirements. Handover Controls an essential component of radio resource management that imposes considerable signaling load on the network. The amount of undesirable signaling load depends directly on the speed of the hand set. In this paper, we propose that an handoff can be initiated by measuring parameters like link quality, location of the mobile and signal power w.r.t neighboring base station using Fuzzy Logic. This method will prevent the unnecessary handoff between the base station in turn reducing the burden of the base station \& preventing traffic conjunction.
\end{abstract}

Key words: Fuzzy Logic, Mobile Networking Handover Controls

\section{INTRODUCTION}

Mobility is the most important features of a Wireless Cellular Communication System. Usually continuous service is achieved by supporting handoff from one cellular system to another. Handoff is the process of changing the channel (frequency, time slot, spreading code or combination of them) associated with the current connection while a call is in progress. It is often initiated either by crossing a cell boundary or by deterioration in the quality of the signal in the current channel. When the mobile moves from the coverage area of one cell to another a new connection from the target cell has to be set up and the connection with the old cell has to be released [2].

Handoff is divided in to two broad categories hard \& soft handoff. In hard handoff current resources are released before the new resources are used where as in soft handoff both existing and the new resources are used during the handoff process.

Regardless of being necessary all types of handovers have common drawback, they decrease the overall system performance due to the signaling load caused by rerouting the ongoing connection to the new cells to where mobile is entering.

As a central component of Radio Resource Management (RRM), Handover Control (HC) is responsible for managing the on going calls even when mobile stations cross the border from one cell in to another [5]. Depending on the diversity used in association with handover mechanisms, they can be categorized as hard, soft, and softer handovers. During the handover process, if the old connection is released before establishing new connection the result is hard handover. Unlike in hard handover, if a new connection is established before the old connection is released then the handover is called a soft handover.

Soft handover is an instinct characteristic of any Code Division Multiple Access (CDMA) - based mobile system in which the same portion of the radio bandwidth is shared simultaneously between the users. All the abovementioned handovers are provided by the third generation mobile system.

Because handover decisions impact directly on the functions \& results of the other entities in RRM, a close cooperation between $\mathrm{HC}$ and these entities is required to help optimize the radio resource allocation with in the radio access [4].

\section{This paper is organized as follows:}

II. The conventional handover are studied.

III. The proposed handover approach is studied by describing its main principles and the basic algorithm

IV. We present the expected result

V. Conclusion.

\section{LITERATURE STUDY (THE STUDY OF CONVENTIONAL HANDOVER)}

Due to increase in the facilities offered by the mobile 
communication system, numbers of mobile users are increasing day to day, hence increasing the burden of the base station. Mobility is the most important feature of a wireless cellular communication system. Usually continuous service is achieved by supporting handoff from one cell to the another. The conventional handover execution criteria rely mainly on the signal quality, traffic distribution and bandwidth but none of the conventional approaches exploit mobile location information when making the handover decision [7]. A mobile location based handover approach has been recently presented in [2]. The underlying assumption of the scheme is that the position and orientation of the mobile can be estimated by the mobile itself, by the base station or $\mathrm{co}$ - operatively by both mobile and the base station. The measurements are used by the base station to predict the next cell for each mobile station. This approach concentrate on predicting the required radio resource for handling the connections during the handover procedures in target cell. This approach does not address the switching and signaling aspects of the handover when utilizing the location assistance data. In predictive channel reservation approach handover is based on the location assistance data, which may be an efficient approach for FDMA (Frequency Division Multiple Access) or TDMA (Time Division Multiple Access) based system. In both TDMA \& FDMA technique a specific bandwidth channel portion is fixed and allocated to the mobile handset for a period of time. Hence multi user interface is not as critical as that of CDMA (Code Division Multiple Access) based system. Where in the frequency bandwidth is shared between the users. Due to the radio network environment \& nature of radio communication the location information cannot alone be reliable for the handover application in an interference - limited system like CDMA[1.9].

In order to over come the above-mentioned drawbacks we propose a technique where in handoff is initiated by considering not only the location information but also the link quality, signal power with respect to neighboring base stations. The link quality mainly consists of

1. Received signal strength

2. $S / /$ ratio

\section{Bit error rate}

The above proposed method can be used both for intra cellular handover as well as inter cellular handover \& also to control micro cellular \& macro cellular regions. The handover initiation is done by measuring the parameters by using Fuzzy Logic. The advantage for using fuzzy logic is to have smooth handover \& to prevent unnecessary handovers.

\section{A briefly introduction to the fuzzy logic used is as follows.}

Fuzzy Logic provides a simple way to arrive at a definite conclusion based upon vague, ambiguous, imprecise, noisy or missing input information. Fuzzy logic approach to control problems mimics how a person would make decisions only much faster $[6,10]$.

Fuzzy logic incorporates a simple rule base IF XAND $Y$ THEN Z approach, which helps in solving the control problems rather than attempting to model a system mathematically. The FL model is empirically based, relying on an operators experience rather than their technical understanding of the system.

\section{Advantages of fuzzy logic approach:}

1. It is inherently robust since it does not require precise noise free input \& output parameters. The output parameter is a smooth function despite variation in the input parameters.

2. Modifications are easier because it processes with respect to user-defined rules.

3. This controller can be processed for multiple input \& multiple output parameters.

4. Controlling the non-linear system using fuzzy logic is much easier compared to the mathematical model.

How fuzzy logic is used in this proposed paper.

1. Chose objectives i.e. parameters and relation between input and output parameter.

2. Using RULE BASED STRUCTURE of fuzzy logic by breaking the problem in to series of "IF X AND $Y$ THENZ".

3. Create $F L$ membership functions like triangular \& trapezoidal shapes that define the values of input \& output parameters.

4. Create pre \& post-processing routines for implementing in software. Processing is done by Fuzzy rule base inference including defuzzification.

\section{THE PROPOSED METHOD FOR HANDOVER IN MOBILE NETWORK USING FUZZY LOGIC}

Due to the rapid growth in number of mobile users usually in urban areas, the cells are spitted in to macro \& micro cells to accommodate large number of users. Usually the micro cells are overlaid with in the macro cell. Assume that the mobile user is moving fast \& 
consequently changes the micro cell very frequently. This causes undesirable signaling load due to inter micro cell handovers. To prevent such situation. The fast moving mobile user can be handed over to the overlying macro cell where there is no need to hand over it as often as it was done for micro cell case. When mobile is accommodating in the micro cell, its location information can be collected in association with other radio resource measurement. According to the 3rd generation mobile networks, the location co-ordinates of the handset can be extracted by the network by mobile handset or by using assistance measurements [3]. The proposed method uses this location information along with link quality and signal power due to neighboring base station.

The most influential criteria in mobile cellular networks are the down link quality (from the access point) \& the up link quality (to the access point) respectively. The link quality $[1,4]$ can be measured in terms of received signal strength, signal to inference ratio and bit error rate. When the quality of the radio link between mobile host and the access point falls below a minimum acceptable value, often due to the mobile host moving away from the access point, a handover to a different access point with better link quality is initiated. For NIHO (Network Initiated Handover) a mobile host periodically measures the link quality to each access point, which currently receives and sends the results to the network.

The signal power due to the neighboring base station will be continuous monitored by the mobile unit \& in turn passes the information to the serving base station. In the 3 rd generation system the location information is available at the radio network controller or Serving Mobile Location Center (SMLC). The SMLC can physically become a part of the RNC either by specifying an interface or by integrating it fully to the RNC. Infact improving the internal intelligence of the radio access network (RAN) by utilizing the location information was one of the primary reasons behind the current architectural solution specified in [3]. The location information may be extracted either periodically or it can be gathered on demand. The location information along with link quality and signal power with respect to neighboring base station are reported Handover Control ( $\mathrm{HC})$ in the base station. The fig [1] depicts the general algorithm employed by our approach. The assumption is that the serving micro cell is completely over laid by a macro cell, to which the mobile is intending to be rerouted. When the mobile is bordering with in the handover area in the micro cell its signal strength is compared with a predefined handover threshold set by the $\mathrm{HC}$, providing the required parameters for the handover decisionmaking. The predefined threshold is based on minimum signal level required to reach the minimum quality of service (QOS) attribute of the on going connection. This also includes a handover margin to avoid the "Ping Pong" effect. Comparing the mobile users with the threshold parameter \& initiating the handoff is primary criteria. The secondary criteria are also met i.e. if there is a radio resource available for the handover execution.

If both the primary \& secondary criteria are not met the handover request is rejected. If the primary criterion is met but if there is no radio resource available at the time of handover, the handover request is put in to a queue with in a pre defined time spam. If the time is expired then the handover request is rejected otherwise it is executed.

\section{FLOW CHART}

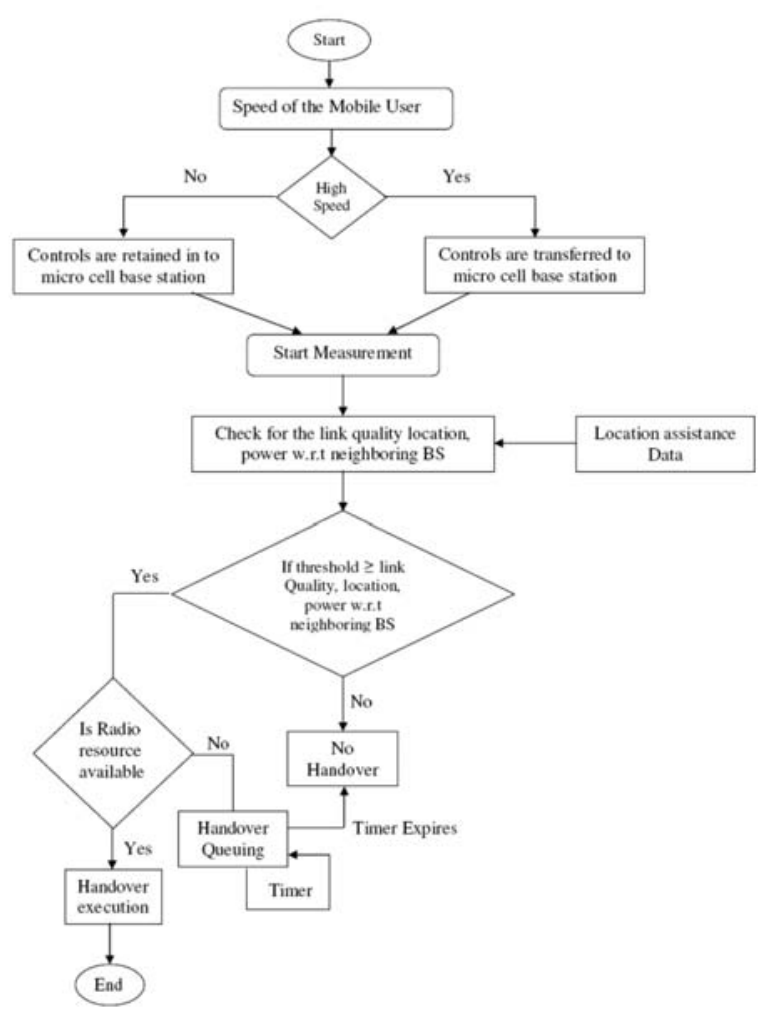

Fig 1

If a mobile is located with in the cellular area with very low link quality, low power w.r.t neighboring base station and with smaller value for location parameter then it is intra cellular handoff. The fig 2 is for intra cellular handover. 


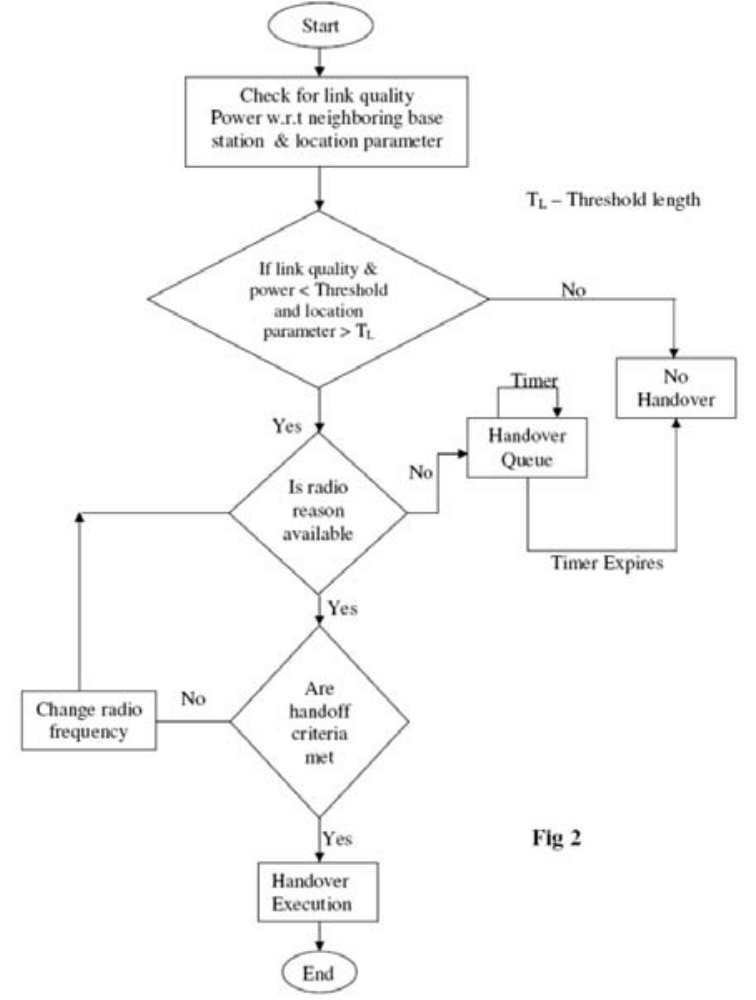

Feg .2

The handover decision may be made based on the measurement results \& the additional mobile positioning parameters, which can be alternatively piggy backed on the handover parameter. As shown when an intra cellular handoff is required, the radio controller considers the location information related to the mobile. In addition to utilizing location information in conjunction with the handover decision-making, the data can also be used when forming the mobility or handover pattern. Based on the latest change of the mobile co-ordinates it can be figured out whether it is fast or slow moving mobile users. By using the mobiles two most recent locations (coordinates) its traveled distance \& speed can also be calculated. In case of Time OF Arrival (TOA) positioning method, the mobile co-ordinates can be determined using the triangulation algorithm. [5]

$$
\mathrm{D}_{\mathrm{i}}=\sqrt{\left(\mathrm{X}_{\mathrm{i}}-\mathrm{X}_{\mathrm{m}}\right)^{2}+\left(\mathrm{Y}_{\mathrm{i}}-\mathrm{Y}_{\mathrm{m}}\right)^{2}+\left(\mathrm{Z}_{\mathrm{i}}-\mathrm{Z}_{\mathrm{m}}\right)^{2}}
$$

Where $(\mathrm{Xi} \mathrm{Yi} \mathrm{Zi})$ are the co - ordinates of the neighboring base station. (Xim Yim Zm) are mobiles co ordinates. $\mathrm{Di}$ - Distance between the handset \& the of arrival circle. Using above information we can get direction of motion of mobile. By using this approach a mobile can be taken for intra cellular handoff or inter cellular handoff \& hence unnecessary handoff can be prevented.

\section{FUZZY RULE BASE INFERENCE}

Fuzzy rule base inference consists of three basic steps \& Defuzzyfication step [].

1. Fuzzy Matching: Calculating the degree to which the input data match the condition of the fuzzy rules i.e. matching degree (min of $\mu i)$

2. Inference: Calculate the rules conclusion based on its matching degree. Conclusion is processed by clipping method.

3. Combination: Combine the conclusion inferred by all fuzzy rules in to a final conclusion.

4. Defuzzification: The centroid (COA) defuzzyfication method calculates the weighted average of a fuzzy set. The result of applying COA defuzzification to a fuzzy conclusion ' $R$ is $A$ ' can be expressed by the formula.

$$
r=\frac{\sum_{i} \mu_{A}\left(r_{i}\right) \times r_{i}}{\sum_{i} \mu_{A}\left(r_{i}\right)} \quad \text { For discrete } r
$$

$$
r=\frac{\int \mu_{A}\left(r_{i}\right) \times r_{i}}{\int \mu_{A}\left(r_{i}\right)} \quad \text { For continuous } r
$$

$S L$ - Signal Strength

$\mathrm{H}-$ high

M - Medium

L- Low

DS- Distance

$\mathrm{N}-\mathrm{Near}$

$\mathrm{F}-\mathrm{Far}$

VF - Very far

P- Power w.r.t neighboring base station

LP - Low Power

HP - High Power

$\mathrm{HO}$ - Handoff Process

$\mathrm{NC}$ - No Change

$\mathrm{C}$ - Change

$\mathrm{SC}$ - Smooth (slow) Change

\section{A. Fuzzy Rule:}

If the serving base Signal strength $(\mathrm{SL})$ is High $(\mathrm{H})$ and the distance (DS) is Near (N) and the Power (P) due to the neighboring base station is Low $(L)$ then the Handoff (HO) process will not be initiated. 


\section{The fuzzy rules for the same can be written as follows.}

If $S L$ is HAND DS is NAND P is LP THEN HO is NC In the same manner for the different possible combination the rules are as follows:

1. If $S L$ is $H A N D$ DS is MF AND $P$ is HP THEN HO is NC

2. If $S L$ is $H A N D D S$ is MF AND $P$ is HP THEN HO is $S C$

3. If $S L$ is $H A N D D S$ is $F A N D P$ is $H P$ THEN HO is $S C$

4. If $S L$ is M AND DS is NAND $P$ is LP THEN HO is $S C$

5. If $S L$ is M AND DS is MF AND $P$ is $L P T H E N H O$ is $S C$

6. If $S L$ is M AND DS is F AND $P$ is LP THEN HO is $S C$

7. If $S L$ is M AND DS is F AND $P$ is LP THEN HO is $S C$

8. If $S L$ is $L A N D D S$ is NAND $P$ is $L P$ THEN HO is NC

9. If $S L$ is $L A N D D S$ is MF AND $P$ is LP THEN HO is $S C$

10. If $S L$ is $L A N D D S$ is $F A N D P$ is $L P$ THEN $H O$ is $S C$

11. If $S L$ is $L A N D D S$ is $F$ AND $P$ is $L P$ THEN HO is $C$

B. Membership function figures:

$\mu$ is Degree of membership function

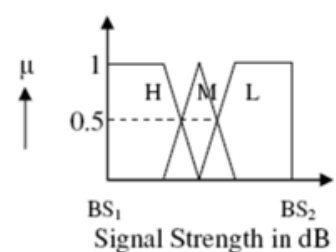

Fig A - Signal Strength Membership Function

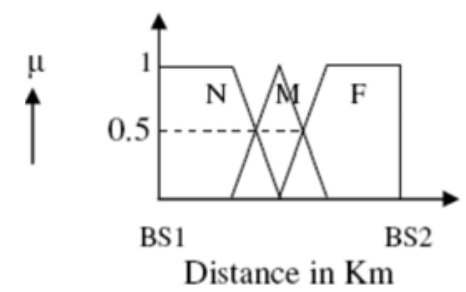

Fig B - Distance Membership Function

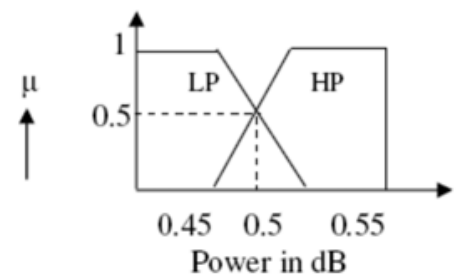

Fig C - Power w.r.t neighboring base station membership function

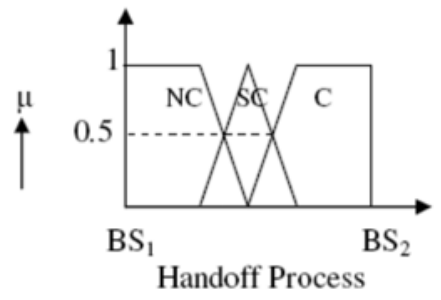

Fig D - Handoff Process membership function

\section{EXPECTED RESULTS}

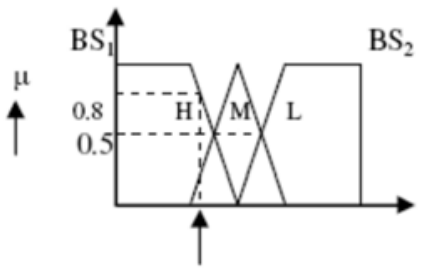

$\mu_{\mathrm{SL}}=0.8$
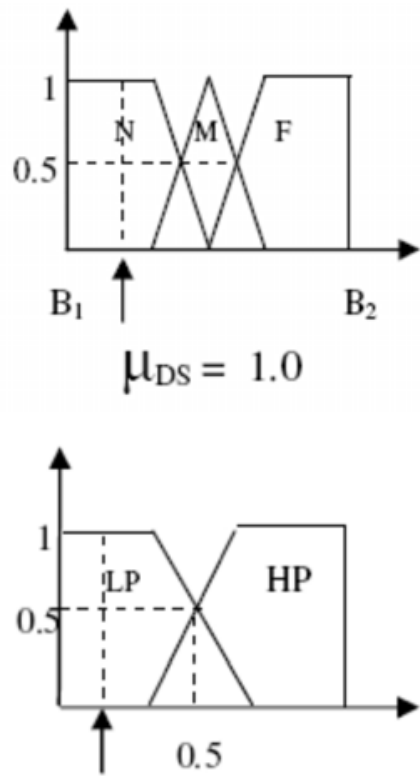

$\mu_{\mathrm{p}}=1.0$

degree if match $=\min (\mu \mathrm{SL}, \mu \mathrm{DS}, \mu \mathrm{P})=\min (0.8,1.0,1.0)$

$$
=0.8
$$

degree of match $=0.8$

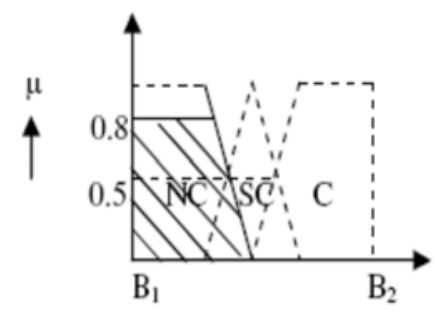

outpu $-1\left(\mu_{\mathrm{SL}}=0.8\right)$ 


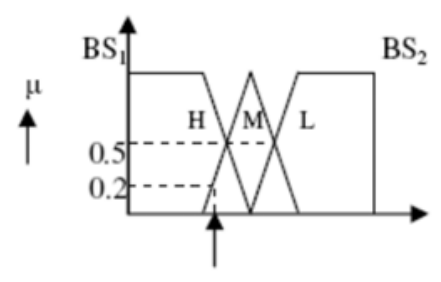

$\mu_{\mathrm{SL}}=0.2$
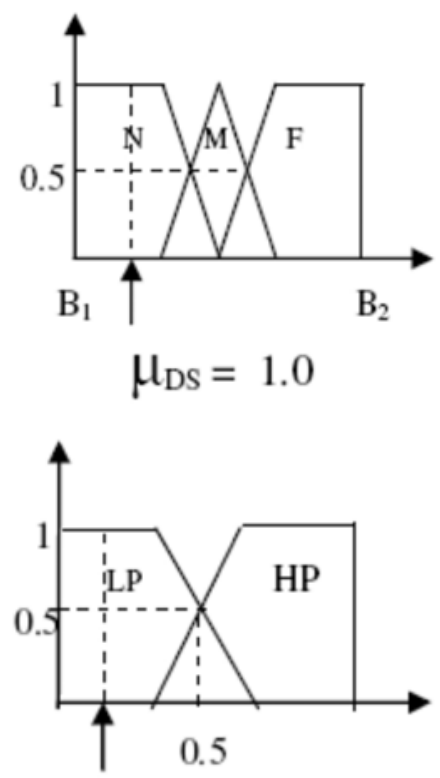

$$
\mu_{\mathrm{p}}=1.0
$$

degree if $\operatorname{match}=\min (\mu S L, \mu D S$, $\mu \mathrm{P})=\min (0.2,1.0,1.0)=0.2$

degree of match $=0.8$

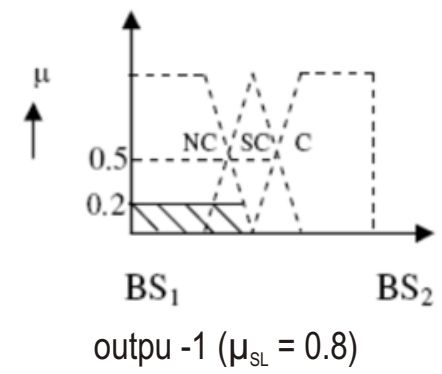

\section{OVERALL RESULT}

Combination of output 1 and output 2 is the final expected result is as shown below with defuzzified value.

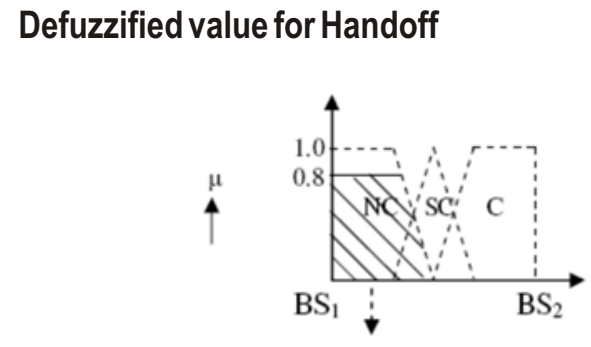

\section{CONCLUSION}

This paper mainly prioritizes smooth handover operation in mobile network communication. Here the handover is initiated by considering the location parameters, line quality \& power w.r.t neighboring base station using fuzzy logic rules. We conclude that mobile positioning will provide valuable information to improve the intelligence of the handover mechanism in cellular network by optimizing the connection route and radio resource allocation policy. This information when combined with link quality information \& power w.r.t neighboring base station will ensure the prevention of unnecessary handover in cellular network. This in turn will reduce the congestion by reducing the burden of the base station.

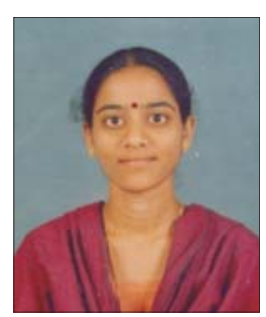

Ms. Sudha P.N. - Assistant Professor, Department of Telecommunication Engineering, K.S.Institute of Technology ,Bangalore.She has published 2 papers in international journals and presented 3 papers in national and international conferences. Her research area is

Mobile Networks. 